INPLASY

PROTOCOL

To cite: Teng et al. Diabetes self care in Malaysia: protocol of an individual participant data meta-analysis of SDSCA studies. Inplasy protocol 202110090. doi:

10.37766/inplasy2021.1.0090

Received: 23 January 2021

Published: 23 January 2021

Corresponding author:

Cheong Lieng Teng

tengcl@gmail.com

Author Affiliation: International Medical University (IMU)

Support: IMU.

Review Stage at time of this submission: Preliminary searches.

Conflicts of interest: None.

\section{Diabetes self care in Malaysia: protocol of an individual participant data meta-analysis of SDSCA studies}

Teng, CL1; Lee, VKMㄹ; Ganeson, M³; Sulaiman, LH4; Bujang, MA55.

Review question / Objective: How is the practice of diabetes self care among adult diabetes patients in Malaysia, and is there any gender or ethnic differences?

Condition being studied: Diabetes self care is a multidimensional construct that can be measured using many rating scales; a commonly used tool is the Summary of Diabetes Self-care Activities (SDSCA) scale. SDSCA assesses the respondents' appropriate action in five domains: diet, exercise, blood sugar testing, foot care and smoking over the past seven days.

Information sources: An extensive literature search using a combination of mesh terms (Self Care; Diabetes Mellitus; Malaysia) and text words (self management, Summary of Diabetes Self-care Activities (SDSCA) scale) will be carried out on electronic databases such as PubMed, Scopus, and MyCite. This will be supplemented by a Google Scholar search using the same search terms mentioned above.

INPLASY registration number: This protocol was registered with the International Platform of Registered Systematic Review and Meta-Analysis Protocols (INPLASY) on 23 January 2021 and was last updated on 23 January 2021 (registration number INPLASY202110090).

\section{INTRODUCTION}

Review question / Objective: How is the practice of diabetes self care among adult diabetes patients in Malaysia, and is there any gender or ethnic differences?
Rationale: This study aims to document the practice and differences (if any) of self care components within SDSCA score of Malaysian diabetes patients. These data will assist health providers and researchers in patient care and clinical research for diabetes adults in Malaysia. 
Condition being studied: Diabetes self care is a multi-dimensional construct that can be measured using many rating scales; a commonly used tool is the Summary of Diabetes Self-care Activities (SDSCA) scale. SDSCA assesses the respondents' appropriate action in five domains: diet, exercise, blood sugar testing, foot care and smoking over the past seven days.

\section{METHODS}

Search strategy: We plan to search bibliographic databases for Malaysian diabetes studies that evaluated self care using the Summary of Diabetes Self-care Activities (SDSCA) scale. Bibliographic search using MESH terms or keywords: Diabetes mellitus, self care, Summary of Diabetes Self-care Activities (SDSCA) scale, Malaysia. There is no limit to the year of publication of included studies.

Participant or population: Adult diabetes patients.

Intervention: Self care score.

Comparator: Subgroups (e.g., gender, ethnicity).

Study designs to be included: Crosssectional studies.

Eligibility criteria: (a) Study participants are adult diabetes patients; (b) Studies conducted in Malaysia; (c) Used the Summary of Diabetes Self-care Activities (SDSCA) scale; (d) Has data on gender or ethnic groups.

Information sources: An extensive literature search using a combination of mesh terms (Self Care; Diabetes Mellitus; Malaysia) and text words (self management, Summary of Diabetes Self-care Activities (SDSCA) scale) will be carried out on electronic databases such as PubMed, Scopus, and MyCite. This will be supplemented by a Google Scholar search using the same search terms mentioned above.
Main outcome(s): Mean (SD) overall score and specific self care practices as measured by SDSCA.

\section{Additional outcome(s): None.}

Data management: References retrieved will be managed using citation manager Endnote 7. The corresponding authors of eligible studies will be contact to request for statistical datasets on SDSCA.

Quality assessment / Risk of bias analysis: The studies identified will then be assessed for methodological validity and relevant data in the included studies were extracted by a pair of investigators and checked by a senior researcher, independently, to minimize potential bias and error as well as to resolve any disagreements. The quality assessment of the published studies will be assessed using Newcastle Ottawa Scale.

Strategy of data synthesis: For quantitative data, where possible, meta-analysis will be performed using MedCalc Statistical Software. For meta-analysis of studies with a continuous measure (comparison of means between treated cases and controls), MedCalc uses the Hedges g statistic as a formulation for the standardized mean difference under the fixed effects model. The heterogeneity statistic is incorporated to calculate the summary standardized mean difference under the random effects model.

Subgroup analysis: (a) Gender; (b) Ethnic groups.

Sensitivity analysis: None.

Language: English.

Country(ies) involved: Malaysia.

Other relevant information: This systematic review will be prepared following PRISMAIPD and MOOSE guidelines.

Keywords: Adult; Diabetes Mellitus; Ethnic Groups; Malaysia; Meta-Analysis; Self Care; Sex Factors. 
Dissemination plans: Publication of manuscript in a journal.

Contributions of each author:

Author 1 - Cheong Lieng Teng - Protocol preparation; literature search and management; statistical analysis; manuscript preparation.

Email: tengcl@gmail.com

Author 2 - Verna Kar Mun Lee - Protocol preparation; literature search and management; manuscript preparation.

Email: verna_lee@imu.edu.my

Author 3 - Malanashita Ganeson - Protocol preparation; literature search and management; manuscript preparation.

Email: malanashita@imu.edu.my

Author 4 - Lokman Hakim Sulaiman Protocol preparation; manuscript preparation.

Email: LokmanHakim@imu.edu.my

Author 5 - Mohamad Adam Bujang Protocol preparation; statistical analysis; manuscript preparation.

Email: adam@crc.gov.my 